\title{
Morfologia do sincrânio do boto-cinza, Sotalia guianensis (P.). van Bénéden) (Cetacea, Delphinidae)
}

\author{
Paulo C. Simões-Lopes
}

${ }^{1}$ Laboratório de Mamíferos Aquáticos, Departamento de Ecologia e Zoologia, Centro de Ciências Biológicas, Universidade Federal de Santa Catarina. Caixa Postal 5102, 88040-970 Florianópolis, Santa Catarina, Brasil. E-mail: Iamaqsl@ccb.ufsc.br

\begin{abstract}
Syncranial morphology of the estuarine dolphin Sotalia guianensis (P.). van Bénéden) (Cetacea, Delphinidae). Morphological and comparative description of the syncranium of Sotalia guianensis (P.). van Bénéden, 1864), including ontogenic aspects and intraspecific variations are presented. The descriptions were based on the examination of 51 complete or partial skulls, kept at the Marine Mammals Lab. Collection from the Departamento de Ecologia e Zoologia, CCB, at the Federal University of Santa Catarina. The sincranium is very homogeneous and manifests its asymmetry in the morphology of the bones, nasal passages and foramina. The rostrum is proportionally narrow in relation to other Delphinidae of the same size. The temporal region is circular in young specimens, but it becomes oval in the adults were it forms a well marked temporal crest. The elliptical foramen of the periotic bone is present both in young and adult individuals. The nasals are bipartite and one may find sutural bones between the frontals. The hamular process of the pterygoids is inflated and somewhat fenestrated. The mandibular symphysis covers from $19-23 \%$ of the dentary length. Each premaxilla and maxilla has 30-35 teeth and each dentary exhibits 30-38 teeth.
\end{abstract}

KEY WORDS. Anatomy; intraspecific variations; ontogeny; skull morphology; small cetaceans.

RESUMO. Apresenta-se uma descrição da morfologia comparada do sincrânio de Sotalia guianensis (P.). van Bénéden, 1864), incluindo suas variações ontogenéticas e intra-específicas. As descrições foram baseadas em 51 crânios completos ou parciais depositados na coleção do laboratório de Mamíferos Aquáticos, do Departamento de Ecologia e Zoologia, CCB, Universidade Federal de Santa Catarina. O sincrânio é bastante homogêneo e manifesta sua assimetria na morfologia dos ossos, passagens nasais e forames. O rostro é proporcionalmente estreito em relação aos outros Delphinidae do mesmo porte. A região temporal é circular em indivíduos jovens, mas se torna oval nos adultos onde se forma uma crista temporal evidente. $O$ forame elíptico do periótico está presente tanto em indivíduos jovens quanto adultos. Os nasais são bipartidos e podem ocorrer ossos suturais entre os frontais. $\mathrm{O}$ processo hamular dos pterigóides é bastante inflado e fenestrado. A sínfise mandibular alcança de 19 de $19-23 \%$ do comprimento do dentário. Cada pré-maxilar e maxilar possui de 30-35 dentes e cada dentário de 30-38 dentes. PALAVRAS-CHAVE. Anatomia; morfologia do crânio; ontogenia; pequenos cetáceos; variações intra-específicas.

De acordo com HershKovitz (1966), cerca de 58 formas nominais foram descritas para o gênero Sotalia Gray, 1866, mas 22 delas foram posteriormente transferidas para o gênero Sousa Gray, 1866. Isto pode explicar parte da instabilidade taxonômica do grupo, mas outras razões também podem ser aduzidas, como a falta de homogeneidade nos critérios comparativos e mesmo a fraca tradição em morfologia e sistemática nos estudos com mamíferos aquáticos.

A partir de Rice (1977) as ditas "formas" ou ecótipos de água doce e marinho de Sotalia tornaram-se, aparentemente, bem estabelecidos e enquadrados em duas distintas subespécies: $S$. $f$. fluviatilis (Gervais, 1853) e S. f. guianensis (P.J. Van Bénéden, 1864). Recentemente, tendo por base estudos de morfometria geomé- trica do crânio (técnica conhecida como "procrustes superimposition"), MonTeiro-FilHo et al. (2002) propõem que estes taxa sejam elevados a categoria de espécie. $S$. guianensis apresenta uma distribuição possivelmente contínua ao longo da costa atlântica, que vai da Nicarágua (SILvA \& BEST 1996) às latitudes da Ilha de Santa Catarina, $27^{\circ} 35^{\prime}$ S, sul do Brasil (SimõEs-Lopes 1988). No estuário amazônico sua distribuição deve entrar em contato com as populações de água doce, mas se desconhece qualquer versão conclusiva a esse respeito, inclusive se existe simpatria.

As descrições osteológicas preliminares devem-se a vAN Beneden \& Gervais (1880), Flower (1883), Miranda-Ribeiro (1936) e Carvalho (1963). Mais recentemente, Casinos et al. (1981) discutiram a morfometria e MENEZEs \& SIMÕEs-Lopes (1996) apresen- 
taram uma descrição simplificada da parte óssea dos membros anteriores. Alves-Junior \& Monteiro-Neto (1999), analisaram as medidas cranianas tendo por base exemplares da costa do Ceará, mas nada acrescentaram com relação à caracterização morfológica, nem com relação aos caracteres diagnósticos. Ávili et al. (2002) apresentaram uma descrição da osteologia geral, mas com várias incorreções de terminologia e localização.

Apresenta-se aqui uma descrição morfológica e comparativa das regiões do sincrânio de $S$. guianensis, incluindo aspectos de ontogenia e comparação com espécies do mesmo porte. Procura-se também reparar eventuais distorções de nomenclatura anatômica.

\section{MATERIAL EXAMINADO E TERMINOLOGIA}

As descrições baseiam-se no exame de 51 espécimes de $S$. guianensis, depositados na coleção de mamíferos, do Departamento de Ecologia e Zoologia, CCB, Universidade Federal de Santa Catarina (UFSC) (Tab. I). Determinadas feições internas do crânio foram obtidas de exemplares jovens ou fetos, onde foi possível desarticular as peças ósseas.

Sempre que possível segue-se a terminologia de FlOwer (1885), Kraglievich (1937), Starck (1967) e Rommel (1990). O termo sincrânio é utilizado de acordo com a concepção de Gregory (1933), onde o neurocrânio, esplancnocrânio e mandíbula são considerados em conjunto, com a finalidade de evitar as freqüentes confusões sobre a porção exclusiva do cranium. Quando analisados pela primeira vez no texto, os nomes dos ossos aparecem acompanhados por seu equivalente em latim entre parênteses, assim como por seus sinônimos.

\section{Sincrânio}

O arranjo craniano seguiu o padrão telescópico dos outros cetáceos como foi proposto inicialmente por MiLLeR (1923), onde os ossos da porção anterior do crânio alongaram-se e escorregam sobre os ossos posteriores, dificultando a visualização destes. A assimetria craniana foi evidente e manifestou-se nas diferenças de tamanho e forma de vários ossos da vista dorsal, aberturas nasais, posição e número dos forames. A abertura da passagem nasal direita é mais estreita que a do lado esquerdo, devido ao formato dos pré-maxilares e da posição relativa do complexo etmóide. Considerando as variações intra-específicas, o sincrânio de $S$. guianensis é extremamente homogêneo (Figs 1-6).

\section{Regiões rostral e palatal}

O rostro ou crânio facial de Sotalia guianensis é proporcionalmente estreito na base em relação a outros Delphinidae do mesmo porte (Lagenorhynchus e Stenella) e sua porção distal é aguda tanto em indivíduos jovens, quanto adultos (Figs 5-6). Os ossos pré-maxilares (os premaxillare) e maxilares (maxilla) são muito alongados posteriormente e chegam a cobrir boa parte dos frontais em vista dorsal (Fig. 7). O pré-maxilar direito quase encosta no nasal em alguns casos, mas isso não ocorre em indivíduos jovens, onde o padrão telescópico é menos pronunciado. Os pré-maxilares fazem contato lateral com os maxilares em toda a sua extensão dorsal e, pela face medial, encontram o vômer (vomer). Este último é um osso impar, em forma de calha, e que serve de apoio para a cartilagem mesorostral.

O palato é achatado e sem sulcos e o vômer aparece apenas numa pequena extensão medial entre os pré-maxilares e maxilares. A região posterior do palato está formada pelos palatinos (os palatinum), que apresentam uma projeção medial posterior em forma de agulha entre os processos hamulares dos pterigóides, afastando-os visivelmente (Fig. 8).

Os forames da região facial apresentam uma distribuição variável. O segundo ramo do nervo trigêmeo ou nervo $\mathrm{V}_{2}$ (vasa infraorbitalia) está alojado num longo canal dentro do maxilar, que só pode ser observado em corte transversal, e emerge em várias aberturas localizadas nos maxilares e pré-maxilares. Esta inferência a partir da configuração anatômica também foi confirmada diretamente através da dissecação dos exemplares UFSC 1052, 1083 e 1175. Tais forames são conhecidos como forames infraorbitais (foramen infraorbitale), que no caso dos cetáceos fogem ao padrão dos outros mamíferos sendo mais numerosos e estando localizados em posição supraorbital (Figs 6-7). Em vista dorsal, cada pré-maxilar possui um único forame na linha que delimita a base do rostro, mas nos maxilares eles variam de quatro a nove, dependendo da quantidade de ramos nervosos terminais. Neste osso eles estão alojados desde a base do rostro até início do processo ascendente. Próximo à base do rostro costuma ocorrer um forame infraorbital maior em cada maxilar. A margem anterior de cada palatino em vista ventral também possui um ou dois orifícios chamados de forames palatinos (foramen palatinum) (Fig. 8).

\section{Regiões orbital e temporal}

A órbita está limitada anteriormente pelos frontais (os frontale) e pelos lacrimais (os lacrimale), este último um osso irregular articulado próximo à base do rostro (Fig. 9). Num exame detalhado do lacrimal em vista ventral, pode-se observar a abertura do canal lacrimal. Dorsal e posteriormente, a órbita é formada pelo frontal, que nesta região só pode ser observado em vista lateral, já que está quase totalmente coberto pelos maxilares e pré-maxilares. O bordo inferior da órbita está limitado pelo arco zigomático, composto em boa parte pelo osso jugal (os jugale, os malare). Como nos outros odontocetos, este é um osso muito delgado que parte do lacrimal sem sutura aparente e vai articular-se, frouxamente, ao processo zigomático do esquamosal (Fig. 1). Com freqüência, este osso é perdido durante as preparações de material e está ausente na maior parte dos exemplares de coleções científicas. Os esquamosais (os squamosale, os temporale) participam da delimitação da órbita posteriormente numa pequena extensão (Figs 1 e 9).

O processo zigomático do esquamosal prolonga-se anterior e dorsalmente, ligando-se por uma terminação cartilaginosa ao processo pós-orbital do frontal. Com este último delimita uma fossa temporal ampla, onde está alojado o musculus temporalis. A região temporal inclui as laterais da caixa craniana e está composta, principalmente, pelos parietais (os parietale) e

Revista Brasileira de Zoologia 23 (3): 652-660, setembro, 2006 

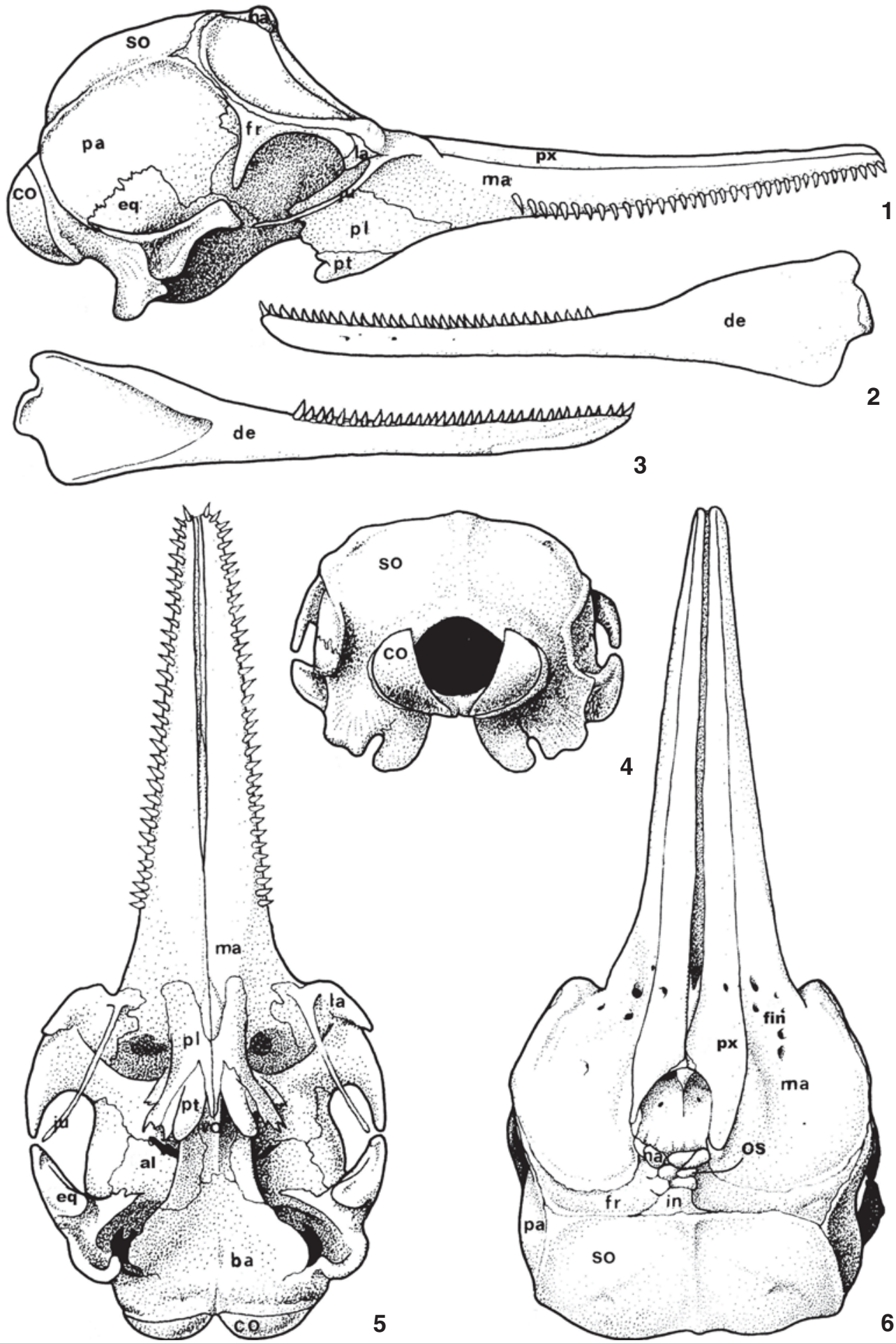

Figuras 1-6. Sincrânio de Sotalia guianensis, vistas: (1) lateral do crânio; (2) lateral do dentário esquerdo; (3) medial do dentário esquerdo; (4) posterior do crânio; (5) ventral do crânio; (6) dorsal do crânio. Abreviaturas: (al) alisfenóide, (ba) basioccipital, (co) côndilo occipital, (de) dentário, (eq) esquamosal, (fin) foramens infraorbitais dorsais, (fr) frontal, (in) interparietal, (ju) jugal, (la) lacrimal, (ma) maxilar, (na) nasal, (os) ossos suturais, (pa) parietal, (pl) palatino, (pt) pterigóide, (px) pré-maxilar, (so) supraoccipital, (vo) vômer.

Revista Brasileira de Zoologia 23 (3): 652-660, setembro, 2006 
esquamosais. Dorsalmente, os parietais penetram em cunha entre o frontal e o supraoccipital. Nos indivíduos neonatos e jovens a região temporal é notadamente convexa ou inflada e não está delimitada por uma crista temporal. Nos animais adultos esta região passa de circular a oval, com maior dimensão ântero-posterior no mesmo sentido do eixo do crânio, e possui uma crista temporal bem marcada (Fig. 9).

\section{Região nasal e teto craniano}

As porções anteriores das passagens nasais são limitadas pelos pré-maxilares e maxilares (este último mais ventral), sendo que o maxilar direito emerge em posição medial, restringindo um pouco mais a passagem nasal direita (Fig. 7). O processo ascendente do pré-maxilar direito é mais largo e quase alcança o nasal em alguns exemplares. Lateral e posteriormente, as passagens nasais estão limitadas pelos frontais (o que pode ser verificado apenas em exemplares muito jovens) e pelo complexo etmóide (os ethmoidale). Os exemplares neonatos e jovens (números 1174, 1178, 1179, 1233) oferecem evidências marcantes de que os ossos deste complexo devem ser considerados separadamente e chamados de mesetmóide (mesethmoideum) e ectetmóides (os ectethmoidale). Do primeiro projeta-se anteriormente a lamina perpendicularis, algo assimétrica, que divide as aberturas nasais. As ossificações pregueadas dos turbinais, que formariam a base do epitélio olfativo, estão completamente ausentes.

Os nasais (os nasale) são pequenos, ligeiramente assimétricos e bipartidos nesta espécie e estão alojados sobre os frontais sem participar da cavidade nasal (Fig. 7). Os frontais formam a maior parte do teto craniano, mas são visíveis apenas numa estreita faixa transversal, que é bastante reduzida em exemplares adultos. Fazem contato entre si medialmente e com um interparietal (os interparietale). Este costuma estar fortemente anquilosado com uma projeção anterior do supraoccipital e em alguns exemplares aparece interposto, completamente, entre os frontais. Esta região do occiput é bastante variável e possui de um a vários ossos suturais (ossa suturarum) alojados entre os frontais e nasais, fato que pode ser observado também em outros mamíferos como os artiodáctilos. Tais ossos podem ser observados também pela parte interna da caixa craniana.

\section{Complexo ósseo dos occipitais}

Esta região resulta da coalescência de quatro ossos endocondrais que circundam o foramen magnum. A participação do basioccipital neste forame está restrita aos estágios iniciais do desenvolvimento ontogênico intra-uterino. Já nos indivíduos neonatos não se pode confirmar sua participação, pois as suturas desapareceram nos estágios precoces. Os exoccipitais (os exoccipitale) portam os côndilos occipitais e ainda estão individualizados nos exemplares neonatos (Fig. 10). Em alguns casos podese observar uma fenestra na junção entre o exoccipital, parietal e supraoccipital, mas ela desaparece completamente já no início da vida. Ventral e lateralmente, junto à sutura com o basioccipital, os exoccipitais portam o foramen hypoglossi, que se abre por um único canal. O supraoccipital constitui a parte superior do foramen magnum. A borda superior deste forame possui uma reentrância em formato de "V" que é mais evidente nos indivíduos jovens, mas permanece em alguns exemplares adultos.

Todo o complexo occipital, que compõem a região posterior do neurocrânio, mostra-se bastante convexo e arredondado nos indivíduos jovens (Fig. 10), mas nos adultos e velhos esta região passa a ser limitada anteriormente por uma crista supraoccipital (nucal ou lambdóidea) alta e inclinada anteriormente. As laterais do supraoccipital também passam a ser vincadas pelas cristas temporais e neste osso pode ocorrer o aparecimento de uma crista medial suave nos estágios mais avançados.

Na porção interna da caixa craniana ocorre a formação de uma projeção óssea a partir do supraoccipital. Esta projeção irá formar, nos estágios mais avançados, um arco ósseo conhecido como tentorium, separando a fossa cerebral e cerebelar.

\section{Região do basicrânio}

Em vista ventral, as cavidades nasais são circundadas pelos palatinos, pterigóides e vômer. A participação dos palatinos nas aberturas nasais pode ser confirmada mais claramente em animais jovens. Os pterigóides (os pterygoideus) são ossos irregulares e separados medialmente por uma projeção posterior em ponta dos palatinos. Os pterigóides possuem um processo hamular inflado e bastante frágil (Fig. 8), que pode ser algo fenestrado em determinados indivíduos. $\mathrm{O}$ vômer desempenha um papel importante na região, separando as aberturas nasais e envolvendo o pré-esfenóide (praesphenoideum) e a base do complexo etmóide. Seu processo posterior laminar possui margens paralelas e separa os processos lamelares dos pterigóides, encobrindo o pré-esfenóide e parte do basiesfenóide (basisphenoideum). O basisfenóide fusiona-se primeiro ao basioccipital e depois ao préesfenóide. Esta última linha sutural ainda pode ser visualizada em alguns exemplares neonatos. Esses ossos formam a maior parte da porção axial do assoalho do neurocrânio.

As porções laterais do basicrânio estão formadas pelos alisfenóides, orbitosfenóides (alae orbitalies), frontais e exoccipitais. Esta é uma região com vários forames por onde saem os demais nervos e artérias cranianas. Com o avanço da idade essas aberturas acabam subdivididas dificultando sua compreensão. Entre o alisfenóide e orbitosfenóide está o forame lacerado anterior por onde saem ramos dos nervos II a VI [segundo RoMmeL (1990) nervos: óptico (II), oculomotor (III), troclear (IV), oftálmico (V) e abducens (VI)]. Atrás do alisfenóide aparece o forame oval, por onde sai um dos ramos do nervo trigêmio $\left(\mathrm{V}_{3}\right)$. A terceira e maior abertura é a do hiato craniano, onde está alojado o complexo periótico-timpânico, que será tratado na próxima sessão. Por esta abertura saem os nervos faciais (VII) e auditivos (VIII). O hiato craniano é amplo em indivíduos jovens, mas fica reduzido nos animais velhos, podendo estar subdividido em várias aberturas menores.

\section{Região periótica}

Nos cetáceos esta região é formada por um complexo ósseo chamado periótico-timpânico, que se encontra separado

Revista Brasileira de Zoologia 23 (3): 652-660, setembro, 2006 

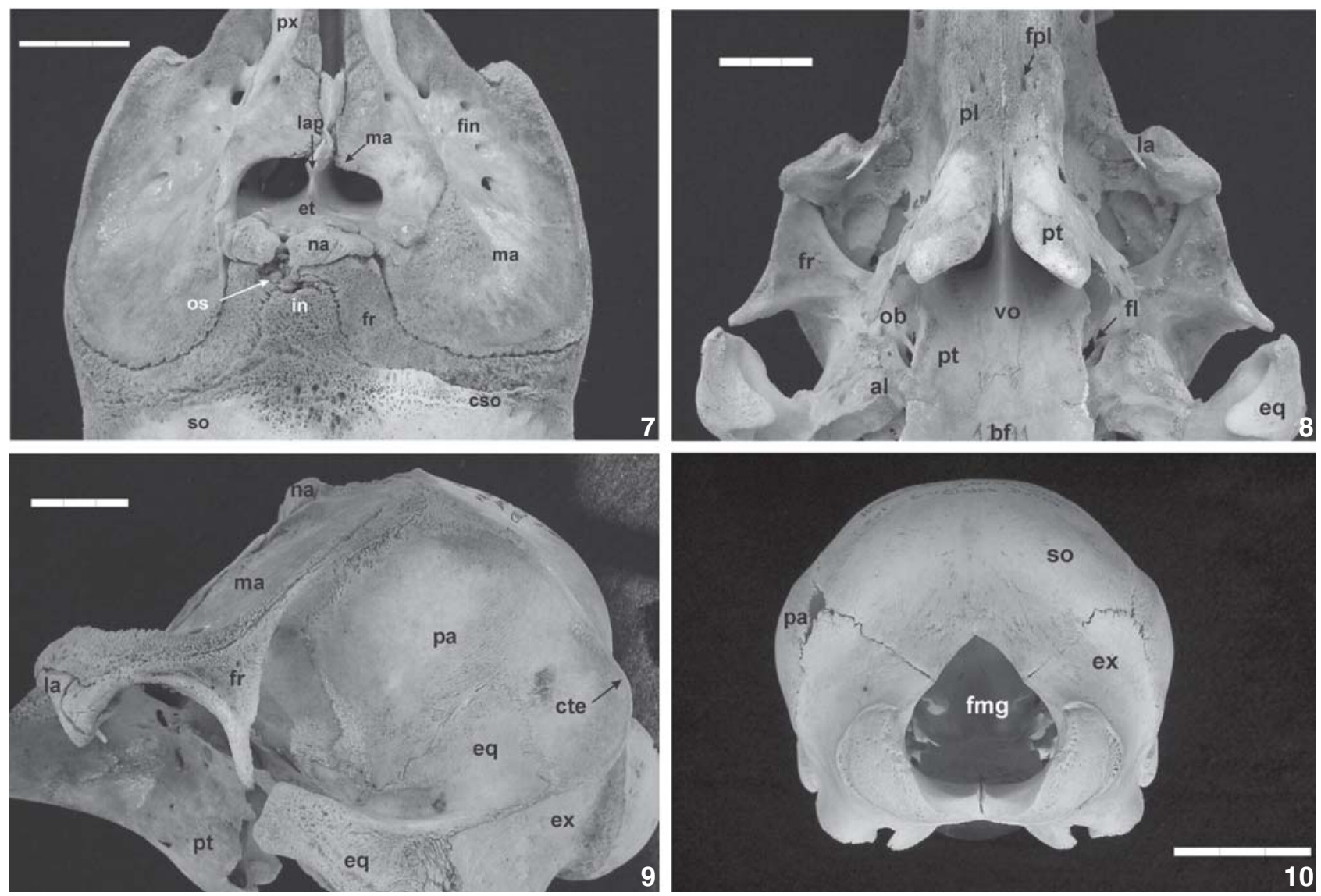

Figuras 7-10. Sotalia guianensis. (7- 9) Exemplar adulto, UFSC 1291: (7) detalhe do teto craniano: (cso) crista supraoccipital, (et) etmóide, (fin) forames infraorbitais dorsais, (fr) frontal, (in) interparietal, (lap) lamina perpendicularis, (ma) maxilar, (na) nasal, (os) ossos suturais, (px) pré-maxilar, (so) supraoccipital; (8) detalhe da base do crânio: (al) alisfenóide, (bf) basiesfenóide, (eq) esquamosal, (fl) forame lacerado, (fpl) forame palatino, (fr) frontal, (la) lacrimal, (ma) maxilar, (ob) orbitosfenóide, (pl) palatino, (pt) pterigóide, (vo) vômer; (9) detalhe das regiões orbital e temporal do crânio, em vista lateral: (cte) crista temporal, (eq) esquamosal, (ex) exoccipital, (fr) frontal, (la) lacrimal, (ma) maxilar, (na) nasal, (pa) parietal, (pt) pterigóide. O osso jugal encontra-se ausente neste exemplar; (10) crânio de exemplar neonato, UFSC 1174, em vista posterior: (ex) exoccipital, (fmg) forame magnum, (pa) parietal, (so) supraoccipital. Escala: $3 \mathrm{~cm}$.

do crânio. Mais precisamente, estes ossos estão alojados abaixo do hiato craniano. O periótico (petrosum) é um osso de substituição, enquanto o timpânico (tympanicum) é um osso de revestimento. Ambos estão ligados por uma sinostose, isto é, sem fusão óssea, podendo ser separados com facilidade.

O timpânico possui poucas características notáveis, além de sua aparência inflada, como as proeminências posterior interna e externa e a projeção anterior levemente arqueada (Figs 11-13). O periótico é mais compacto e seu processo posterior, junto à sutura com o timpânico, torna-se cada vez mais rugoso com o avanço da idade. Seu forame elíptico está aberto tanto em indivíduos jovens, quanto adultos (Fig. 11). A porção coclear segue o padrão dos Delphinidae, com o fundus do meato auditivo interno contendo o foramen singulare e a abertura interna do aquaeductus Fallopii. Externamente ao fundus do meato au- ditivo interno estão as aberturas do aquaeductus cochleae, do ductus endolymphaticus e do foramen rotundum (Fig. 13).

\section{Mandíbula}

Cada dentário (dentale) possui um processo anterior (processus alveolaris) que porta os dentes. A extremidade anterior deste processo é delgada e está levemente voltada dorsalmente nesta espécie (Figs 2-3). Nela emergem 4 a 7 forames mentonianos, que transmitem o ramo mandibular do trigêmio $\left(n . V_{3}\right)$. O número destes forames varia entre ossos dentários de um mesmo indivíduo. Cada dentário está unido anteriormente por uma sínfise mandibular pouco consistente, que alcança em torno de 19 e $23 \%$ do comprimento do dentário $(\mathrm{n}=51)$. A porção posterior o dentário apresenta-se aplanada, possuindo apenas uma crista coronóide (processus coronoideus), 
algo rugosa e inclinada posterior e lateralmente, onde se insere o músculo temporal. A articulação com o crânio se dá pelo processus condyloideus, cuja borda torna-se bastante pronunciada e irregular com o avanço da idade. Em vista medial, abre-se um amplo forame mandibular (foramen mandibularis), que aloja os nervos e vasos mandibulares.

\section{Dentição}

Os canais dentários da maxila e pré-maxila abrigam de 30 a 35 dentes $(n=43)$ de cada lado, sendo que apenas um ou dois pares, muito reduzidos, estão implantados no pré-maxilar. Contagens inferiores são geralmente originárias de exemplares com a extremidade do rostro desgastada. Os alvéolos dentários dos neonatos ainda não estão delimitados, mas o processo de individualização dos alvéolos inicia bem cedo no desenvolvimento ontogenético. Cada dentário também porta de 30 a 38 dentes $(n=43)$, que ocluem com os superiores, quando a mandíbula se fecha.

Os dentes são cônicos e possuem o ápice voltado para a face lingual. Os maiores dentes alcançam em torno de $19 \mathrm{~mm}$ de comprimento total. A coroa é lisa e de seção transversal levemente elíptica. Nos indivíduos velhos pode estar desgastada obliquamente ou mesmo inteiramente destruída pela oclusão dentária. Podem ocorrer casos raros de coroas duplas ou com separação incipiente. As coroas estão separadas da raiz por um colo dentário bem definido em animais adultos. $\mathrm{O}$ maior diâmetro nessa região está no sentido labial-lingual e fica entre 4,3 e 5,3 $\mathrm{mm}$ e o diâmetro menor entre 3,8 e $4,3 \mathrm{~mm}$. A raiz única é longa e sua extremidade costuma estar voltada em direção caudal.

\section{DISCUSSÃO}

Os gêneros Sotalia, Sousa e Steno Gray, 1846 já foram agrupados na família Stenidae, tendo por base a anatomia dos seios aéreos (Fraser \& PURVes 1960), ou mais freqüentemente na subfamília Steninae, dentro da família Delphinidae, de acordo com caracteres do esqueleto (BARNes et al. 1985). No entanto, a análise cladística das sequências de citocromo $b$ indica um agrupamento diferente, onde Sousa aparece claramente separado dos outros dois gêneros (LeDuc et al. 1999).

Considerando a morfologia do sincrânio, os três gêneros assemelham-se na forma globosa do neurocrânio, nas fossas temporais amplas e quase circulares, e na base muito estreita do rostro (ver Miyazaki \& Perrin 1994: para Steno; Ross et al. 1994: para Sousa). No entanto, as diferenças morfológicas entre Steno e Sotalia são evidentes em todos os estágios ontogenéticos. O primeiro possui dentes grandes com rugosidades longitudinais e estas são mais pronunciadas nos juvenis (MiYazaki \& Perrin 1994).

Os gêneros Sotalia e Sousa possuem a morfologia dos dentes e do crânio muito semelhantes. Miranda-Ribeiro (1936), diferenciou Sotalia dos outros gêneros com base na separação medial dos pterigóides, mas Pilleri \& GIRL (1972) ressaltaram que esta característica aparece também em Sousa. Esta aparente discordância deve-se ao fato de os dois gêneros se acharem reunidos nesta época. Hershrovitz (1966) ainda listou as espécies de Sousa (S. chinensis, S. plumbea e $S$. teuszii) incluídas em Sotalia, porém tal separação já havia sido proposta por Fraser \& PURVEs (1960). Ross et al. (1994) descrevem Sousa com os hâmulos peterigóides côncavos em sua superfície lateral e uma crista longitudinal ventral, diferindo do processo hamular lateralmente convexo, inflado, divergente e geralmente fenestrado de Sotalia.

KasuYa (1973) havia reunido tentativamente Sotalia, Sousa e Cephalorhynchus Gray, 1846 na subfamília Sotaliinae, considerando a morfologia do complexo periótico-timpânico. Para esse autor, os três gêneros compartilhariam um sulco mediano e uma quilha ventral no timpânico, além do forame elíptico, segundo ele, inteiramente fechado. Os dois primeiros caracteres conferem em certa medida, mas ao contrário do que foi mencionado por ele o forame elíptico de $S$. guianensis estava presente em todos os exemplares $(\mathrm{n}=33$ ), tanto nos neonatos e jovens quanto nos adultos. Esta discrepância pode estar relacionada à pequena amostra utilizada por KASUYA (1973): S. guianensis (n = 1), S. fluviatilis $(\mathrm{n}=1)$, Cephalorhynchus sp. $(\mathrm{n}=1)$, Sousa teuszii (Kukenthal, 1892) ( $\mathrm{n}=1)$.

A marcada assimetria da região nasal de $S$. guianensis, com o maxilar direito emergindo em posição medial e restringindo ainda mais a passagem nasal direita, difere do que ocorre em vários outros Delphinidae como Tursiops Gervais, 1855, Steno, Globicephala Lesson, 1828, Pseudorca Reinhardt, 1862 e Orcinus Fitzinger, 1860, mas assemelha-se ao arranjo apresentado por Stenella attenuata (Gray, 1846) e S. frontalis (G. Cuvier, 1829).

Os pterigóides divergentes e separados medialmente, e a fossa temporal ampla diferenciam Sotalia tanto de Stenella attenuata quanto de $S$. frontalis. Nestas duas últimas espécies os pterigóides aparecem paralelos e justapostos medialmente e a fossa temporal é proporcionalmente menor (ver PERRIN \& HoHN 1994: para S. attenuata e PeRRIN et al. 1994a: para S. frontalis). Em S. guianensis a maior dimensão da fossa temporal está disposta no mesmo sentido ântero-posterior do eixo do crânio, enquanto em $S$. frontalis ela é oblíqua a este eixo.

O palato achatado e sem sulcos diferencia Sotalia do gênero Delphinus Linnaeus, 1758 e de algumas espécies do gênero Stenella Gray, 1866: S. longirostris (Gray, 1828), S. clymene (Gray, 1846) e S. coeruleoalba (Meyen, 1833) (ver Evans 1994: para Delphinus, Perrin \& Gilpatrick 1994: para S. longirostris, PerRIN \& Mead 1994: para S. clymene, PerRin et al. 1994b: para S. coeruleoalba). Em vista dorsal, o rostro de Sotalia difere tanto de Delphinus quanto de $S$. clymene, já que nestas espécies ocorre um notável arqueamento dos pré-maxilares com freqüente fusão medial.

Tentativas de comparar a morfometria (caracteres métricos) e morfologia craniana (merísticos) de exemplares marinhos e fluviais do gênero Sotalia sugerem que os exemplares marinhos seriam maiores que os fluviais (Mitchell 1975). Silva \& Best (1994) referiram-se ao maior número de dentes da mandíbula dos exemplares marinhos como um caráter útil na diagnose, mas não se deve desconsiderar o fato de que o maior número de dentes pode simplesmente aparecer como uma con- 


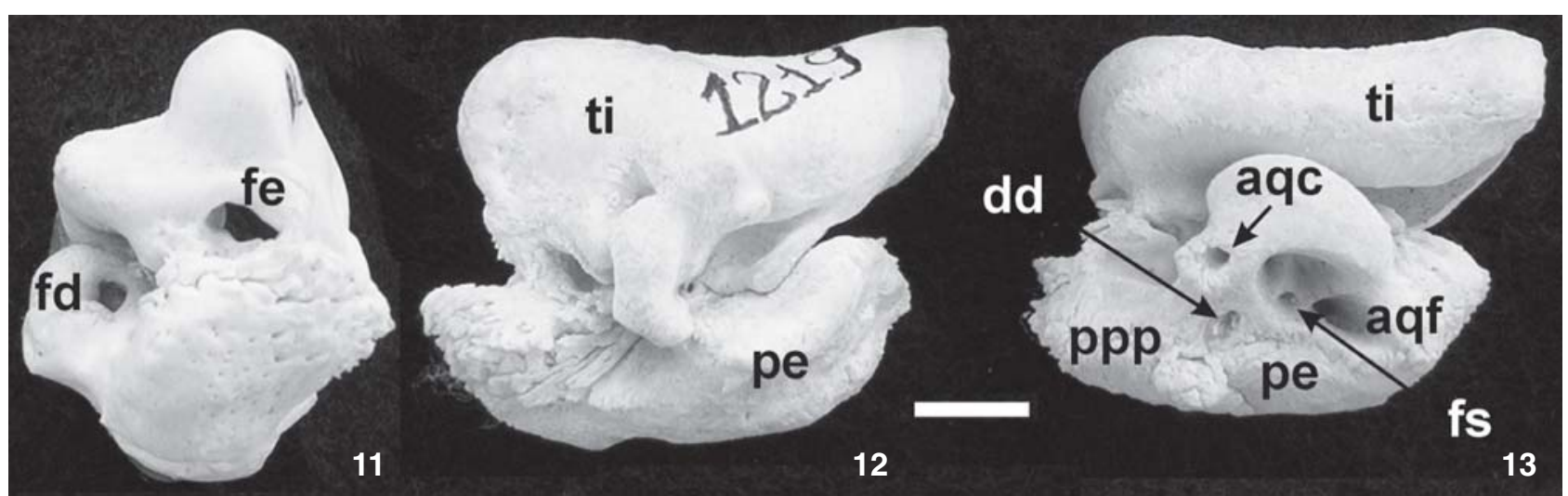

Figuras 11-13. Complexo periótico-timpânico de Sotalia guianensis, exemplar adulto, UFSC 1219. (11) Vista posterior: (fd) forame rotundo, (fe) forame elíptico; (12) vista lateral: (pe) periótico, (ti) timpânico; (13) vista medial: (aqc) aquaeductus cochleae, (aqf) aquaeductus Fallopii; (dd) ductus endolymphaticus, (fs) forame singular, (pe) periótico, (ppp) processo posterior do periótico, (ti) timpânico. Escala: $1 \mathrm{~cm}$.

seqüência do maior tamanho. A mesma estratégia foi utilizada na busca de diferenças populacionais entre os exemplares marinhos (Casinos et al. 1981, Alves-Junior \& Monteiro-Neto 1999), porém os aspectos de morfologia propriamente ditos não foram abordados. Apenas Monteiro-Filho et al. (2002), tendo por base a morfometria geométrica, apresentaram um avanço substancial à questão e propuseram um status de espécie às subespécies propostas por Rice (1977).

Estudos de morfologia comparada do sincrânio que incluam os caracteres diagnósticos e as variações ontogenéticas ou intraespecíficas foram relegados ao segundo plano na maior parte dos estudos. Alves-Junior \& Monteiro-Neto (1999: 92-94), listaram alguns caracteres relativos à caixa craniana e aparato alimentar dos exemplares marinhos, mas tais itens não podem ser considerados exclusivos ou diagnósticos e sim comuns a todos os Delphinidae. CarvalHo (1963) referiu-se brevemente a morfologia dos dentes e alvéolos dentários de Sotalia guianensis e seus valores de diâmetro maior e menor foram levemente inferiores aos aqui apresentados. Alves-Junior \& Monteiro-Neto (1999), relataram que os dentes eram retos na dimensão longitudinal, considerando os exemplares marinhos da costa do Ceará, mas isto não confere com o ápice voltado para a face lingual que foi descrito tanto por Carvalho (1963) como no presente estudo. Relataram também que os dentes estavam implantados nos ossos maxilar e dentário, sem fazer referência aos dentes dos pré-maxilares. Esta falta foi posteriormente corrigida por Ávila et al. (2002) para os mesmos exemplares da costa do Ceará. Alves-Junior \& Monteiro-Neto (1999) e Ávila et al. (2002) apresentaram uma contagem menor nos dentes mandibulares, quando comparadas às linhas dentárias superiores, mas isto difere do que foi constatado na literatura (CARVAlho 1963, Bittencourt 1984, Pinedo et al. 1992, SiLVA \& Best 1994, 1996). Não fica claro se esta é uma característica regional ou se é devida ao desgaste na extremidade da mandíbula.

Fotografias ou desenhos do sincrânio dos exemplares de água doce e que permitam confrontação com exemplares costei- ros são bastante raros na literatura. vAN BéNÉDEN \& GERVAIS (1880: prancha XLI, figs 6-17) apresentaram uma gravura de boa qualidade do sincrânio de Sotalia pallida (Gervais, 1855) [= Delphinus pallidus], sinônimo junior de S. fluviatilis (Gervais, 1853). Numa comparação direta podem-se reconhecer diferenças notáveis na região do basicrânio, onde o processo posterior laminar do vômer é mais largo (amplo) do que cada um dos os processos lamelares dos pterigóides. Isto também fica evidente na fotografia do exemplar no. 047 que foi apresentada por Silva \& BEST (1996: fig. 2). Ocorre exatamente o inverso nos exemplares de $S$. guianensis do sul e sudeste do Brasil, onde o processo posterior laminar do vômer é mais estreito do que os processos lamelares dos pterigóides (Fig. 8). Cabe salientar aqui que as principais tendências evolutivas entre os odontocetos e a maior parte das modificações importantes neste grupo estão relacionadas ao topo do crânio, estejam elas associadas à anatomia facial das passagens nasais ou as condições encontradas nos ossos (exceção feita ao isolamento dos ossos periótico-timpânicos). Dentre estas estão a concavidade dorsal do cranium, a elevação e a assimetria do vértice craniano, a presença de forames nos pré-maxilares e a expansão da face com encurtamento da região intertemporal ("telescoping") (Fordyce \& Barnes 1994, Messenger \& McGuire 1998). Portanto, qualquer modificação na região do basicrânio é evidentemente importante e incomum, merecendo ser investigada numa amostra maior para detectar possíveis variações populacionais.

\section{AGRADECIMENTOS}

A Leandro L. de Sousa, Daniela Fettuccia, Pedro V. de Castilho e aos consultores anônimos.

\section{REFERÊNCIAS BIBLIOGRÁFICAS}

Alves-Junior, T.T. \& C. Monteiro-Neto. 1999. Caracterização morfológica e morfométrica do boto-cinza, Sotalia fluviatilis (Gervais, 1853) da costa do estado de Ceará, Brasil. Arquivos de Ciências do Mar, Fortaleza, 32: 89-101. 
Ávila, F.J.C. \& T.T. Alves-Junior; C.L. Parente; L. De A. L. Vaz \& C. Monteiro-Neto. 2002. Osteologia do Boto-cinza, Sotalia fluviatilis Gervais, 1853, da costa do estado do Ceará, Brasil. Arquivos de Ciências do Mar, Fortaleza, 35: 145-155.

BARNeS, L.G.; D.P. Domming \& C.E. RAy. 1985. Status of studies on fossil marine mammals. Marine Mammal Science, Lawrence, 1 (1):15-53.

BitTenCourT, M.L. 1984. Contribuições à identificação dos cetáceos ocorrentes na costa e águas interiores do Brasil. Arquivos de Biologia e Tecnologia, Curitiba, 27 (4): 529-547.

Carvalho, C.T. DE. 1963. Sobre um boto comum no litoral do Brasil. Revista Brasileira de Biologia, Rio de Janeiro, 23 (3): 263-276.

Casinos, A.; F. Bisbal \& S. Boher. 1981. Sobre tres exemplares de Sotalia fluviatilis del Lago Maracaibo (Venezuela) (Cetacea, Delphinidae). Publicaciones del Departamento de Zoologia de Barcelona, Barcelona, 7: 93-96.

Evans, W.E. 1994. Common dolphin, white-bellied porpoise Delphinus delphis Linnaeus, 1758, p. 191-224. In: Sam H. Ridgway \& Sir Richard Harrison (Ed.). Handbook of Marine Mammals. London, Academic Press, XX+416p.

FLowER, W.H. 1883. On the characters and divisions of the family Delphinidae. Proceedings of the Zoological Society of London, London, 32: 466-513.

FLower, W.H.1885. An Introduction to the Osteology of the Mammalia. London, Macmillan, $3^{\text {rd }}$ ed. [reprinted by Asher, Amsterdam,1966], 344p.

FordyCE, R.E. \& L.G. BARNes. 1994. The evolutionary history of whales and dolphins. Annual Review of Earth and Planetary Sciences, Palo Alto, 22: 419-455.

Fraser, F.C.\& P.E. Purves. 1960. Hearing in cetaceans: evolution of the accessory air sacs and the structure and function of the outer and middle ear in recent cetaceans. Bulletin of the British Museum of Natural History, London, 7: 1-140.

Gregory, W.K. 1933. Fish skulls: a study of the evolution of natural mechanism. Transactions of the American Philosophical Society, Philadelphia, 23 (2): 75-481.

Hershrovitz, P. 1966. Catalog of living whales. United States National Museum Bulletin, Washington, 246: 1-259.

KaSUYA, T. 1973. Systematic consideration of recent toothed whales based on the morphology of tympano-periotic bone. Scientific Reports of Whales Research Institute, Tokyo, 25: 1-103.

Kraglievich, L. 1937. Manual de paleontologia Rioplatense: osteologia comparada de los mamiferos. Montevideo, El Siglo Ilustrado, 141p.

LeDuc, R.G.; W.F. Perrin \& A.E. Dizon. 1999. Phylogenetic relationship among delphinid cetaceans based on full cytocrome $b$ sequences. Marine Mammal Science, Lawrence, 15: 619-648.

MENEZES, M.E. \& P.C. SiMÕES-LOPES. 1996. Osteologia e morfologia da aleta peitoral da forma marinha de Sotalia fluviatilis (Cetacea-Delphinidae) no litoral do Brasil. Estudos de Bio- logia, Curitiba, 4 (40): 23-31.

Messenger, S.L. \& J. McGuire. 1998. Morphology, molecules and phylogenetics of cetaceans. Systematic Biology, Oslo, 47 (1): 90-124.

Miller, G.S. 1923. The telescoping of the cetacean skull. Smithsonian Miscelaneous Collections, Washington, 76 (5): 162.

Miranda-Ribeiro, A. De. 1936. Notas cetológicas dos gêneros Steno, Sotalia e Stenopontistes. Boletim do Museu Nacional do Rio de Janeiro, Rio de Janeiro, 12 (1): 3-23.

Mitchell, E. 1975. Review of Biology and Fisheries for smaller cetaceans. Journal of the Fisheries Research Board of Canada, Ottawa, 32: 875-1240.

Miyazaki, N. \& W.F. Perrin. 1994. Routh-toothed dolphin - Steno bredanensis (Lesson, 1828), p. 1-22. In: S.H. RidGWAY \& S.R. HARRISON (Ed.). Handbook of marine mammals. London, Academic Press, XX+416p.

Monteiro-Filho, E.L.A.; L.R. Monteiro \& S.F. dos Reis. 2002. Skull shape and size divergence in dolphins of the genus Sotalia: a tridimensional morphometric analysis. Journal of Mammalogy, Washington, 18 (1): 125-134.

Perrin, W.F. \& J.W. Gilpatrick Jr. 1994. Spinner dolphin - Stenella longirostris (Gray, 1828), p. 99-128. In: S.H. RIDGWAY \& S.R. HARRISON (Ed.). Handbook of marine mammals. London, Academic Press, XX+416p.

Perrin, W.F. \& A.A. Hohn. 1994. Pantropical spotted dolphin Stenella attenuata, p. 71-98. In: S.H. RIDGWAY \& S.R. HARRISON (Ed.). Handbook of marine mammals. London, Academic Press, XX+416p.

Perrin, W.F. \& J.G. Mead. 1994. Clymene dolphin - Stenella clymene (Gray, 1846), p. 161-172. In: S.H. RIDGWAY \& S.R. HARRISON (Ed.). Handbook of marine mammals. London, Academic Press, XX+416p.

Perrin W.F.; D.K. Caldwell \& M.C. Caldwell.1994a. Atlantic spottede dolphin - Stenella frontalis (G. Cuvier, 1829), p. 173-190. In: S.H. RidgwaY \& S.R. HaRRISON (Ed.). Handbook of marine mammals. London, Academic Press, XX+416p.

Perrin W.F.; C.E. Wilson \& F.I. Archer II. 1994b. Striped dolphin - Stenella coeruleoalba (Meyen, 1833), p. 129-160. In: S.H. RidghaY \& S.R. HARRISON (Ed.). Handbook of marine mammals. London, Academic Press, XX+416p.

Pilleri, G. \& M. Girl. 1972. Contribution to the knowledge of cetaceans of Pakistan with particular reference to the genera Neomeris, Sousa, Delphinus and Tursiops and description of a new chinese porpoise (Neomeris asiaeorientalis). Investigations on Cetacea, Paciano, 10: 129-135.

Pinedo, M.C.; F.C.W. Rosas \& M. Marmontel. 1992. Cetáceos e pinípedes do Brasil. Manaus, UNEP/FUA, X+213p.

RICE, D.W. 1977. A list of Marine Mammals of the World. NOAA, Technical Report NMFS Series, Seattle, 711: 1-15

Rommel, S. 1990. Osteology of the Bottlenose Dolphin, p. 2949. In: S. Leatherwood \& R.R. Reeves (Eds). The bottlenose dolphin. San Diego, Academic Press, XVIII+653p. 
Ross, G.J.B.; G.E. Heinsohn \& V.G. Cockroft. 1994. Humpback Dolphins - Sousa chinensis (Osbeck, Lesson, 1828), Sousa plumbea (G. Cuvier, 1829) and Sousa teuszii (Kukenthal, 1892), p. 23-42. In: S.H. Ridgway \& S.R. HARrison (Ed.). Handbook of marine mammals. London, Academic Press, $\mathrm{XX}+416 \mathrm{p}$.

Silva, V.M.F. DA \& R.C. Best. 1994. Tucuxi - Sotalia fluviatilis (Gervais, 1853), p. 43-69. In: S.H. RidgwaY \& S.R. HARrison (Ed.). Handbook of marine mammals. London, Academic Press, $\mathrm{XX}+416 \mathrm{p}$.

SILVA, V.M.F. DA \& R.C. BEst. 1996. Sotalia fluviatilis. Mammalian
Species, Northampton, 527: 1-7.

SimÕEs-LoPES, P.C. 1988. Ocorrência de uma população de Sotalia fluviatilis (Gervais, 1853) (Cetacea-Delphinidae) no limite sul de sua distribuição, Santa Catarina, Brasil. Biotemas, Florianópolis, 1 (1): 57-62.

STARCK, D. 1967. Les Crâne des Mammifères, p. 405-549. In: P.P. Grassé (Ed.). Traité de Zoologie, anatomie, systématique, biologie. Tome XVI (Fascicule I). Mammifères: tèguments et squelettes. Paris, Ed. Masson et Cie. Éditeurs, VIII+1162p. van Bénéden, P.J. \& P. Gervais. 1880. Osteographie des Cetaces, Vivants et Fossiles. Paris, Arthus Bertrand, 634p.

Recebido em 16.IX.2005; aceito em 09.VIII.2006. 